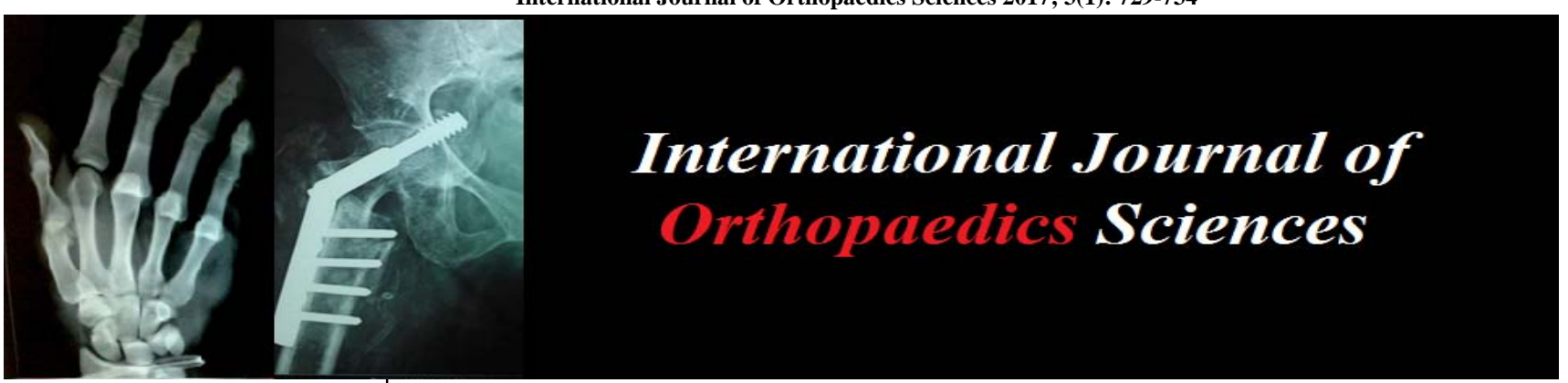

ISSN: $2395-1958$

IJOS 2017; 3(1): 729-734

(C) 2017 IJOS

www.orthopaper.com

Received: 17-11-2016

Accepted: 18-12-2016

Dr. Kosalaraman Padmanaban Associate Professor,

Department of Orthopaedics, Coimbatore Medical College

Hospital, Coimbatore,

Tamilnadu, India

Dr. Balamurugan Palanisamy Assistant Professor,

Department of Orthopaedics, Coimbatore Medical College

Hospital, Coimbatore,

Tamilnadu, India

Dr. Vivekanandhan Ramasamy Assistant Professor, Department of Orthopaedics, Coimbatore Medical College Hospital, Coimbatore,

Tamilnadu, India

Dr. Vijay Krishnan Arcot Subramaniyan Junior Resident Department of Orthopaedics, Coimbatore Medical College

Hospital, Coimbatore,

Tamilnadu, India

Correspondence

Dr. Kosalaraman Padmanaban Associate Professor,

Department of Orthopaedics, Coimbatore Medical College

Hospital, Coimbatore,

Tamilnadu, India

\title{
Analysis of percutaneous tension band wiring for patellar fractures
}

\section{Dr. Kosalaraman Padmanaban, Dr. Balamurugan Palanisamy, Dr. Vivekanandhan Ramasamy and Dr. Vijay Krishnan Arcot Subramaniyan}

DOI: http://dx.doi.org/10.22271/ortho.2017.v3.i1k.107

Abstract

Aim: Patella fractures are more common and account for approximately $1 \%$ of all fractures ${ }^{1}$. The most common method of fixation is a modified tension band wiring by using $2 \mathrm{k}$ wires through a midline longitudinal incision which has its own complications. We analyzed the outcome of percutaneous tension band wiring which has less complication rate than open tension band wiring.

Materials and Methods: Our study was a prospective study conducted in Department of Orthopaedics, Coimbatore medical college during March 2015 to December-2016. We analyzed the clinical and radiological outcome of transverse patellar fractures in 20 patients treated with percutaneous tension band wiring. There were 14 men and 6 women aged 22 to 50 (mean 36 years) in our study. Pain, operating time, mobility, functional score, and complications were evaluated.

Results: Operative time and blood loss in percutaneous tension band wiring is much less compared with open tension band wiring. This technique both clinically and radiologically showed rapid bony union. Lysholm knee score that was used in our study is more with percutaneous tension band wiring. Mean operating time was 30 (range 20 to 50) minutes. The mean follow up period was 8 months (range 6 to 12) months. At the latest follow-up, all patients had good results with painless knee motion, no quadriceps wasting and no subjective disability. Total range of motion was excellent at an average of 142.4 degrees of knee flexion. There were no complications like infection, limitation of knee motion, loss of reduction, implant breakage. All patients had radiological union at eight weeks.

Conclusion: As the percutaneous tension band wiring is done without a wide skin incision, patient satisfaction is more. This technique provide stable fixation, allows early mobilization by minimizing injury to extensor mechanism and reduce cosmetic problem in scar. Hence we conclude percutaneous tension band wiring is a safe and alternative procedure to open tension band wiring

Keywords: Patella fractures, percutaneous wiring, functional outcome

\section{Introduction}

Patella, the largest sesamoid bone is located in the extensor apparatus of the knee which is formed by quadriceps muscle and its tendon, patella, patellar tendon and tibial tuberosity. Anatomical features includes the cranial base, extra articular caudal apex as well as anterior extra articular and posterior articular surfaces. Fracture patella accounts for $1 \%$ of all skeletal fractures. Most are transverse fractures involving middle third caused by indirect violence due to forceful contraction of the quadriceps with knee in flexed position. The common age incidence is between 20 to 50 years and twice common in males than in females. Direct injuries usually result in comminuted fracture. Patient history typically includes a direct blow to the patella, a fall from standing height, or a near fall with forceful contraction of the quadriceps on a partially flexed knee. On physical examination, displaced patella fractures typically present with an acute hemarthrosis and a tender, palpable defect between the fracture fragments. The absence of a large effusion in the presence of palpable bony defect should raise concern for associated retinacular tears. Competence of the extensor mechanism must be assessed by asking the patient to perform a straight-leg raise or extend a partially flexed knee against gravity. It is critical to note, however, that the patient's ability to extend the knee does not rule out a patella fracture but rather it suggests that the continuity of the extensor mechanism is maintained via an intact retinacular sleeve. 
The degree of fragment displacement is generally representative of occult Injury to adjacent soft tissue envelope. The fracture pattern may also depend upon age, bone quality and degree of knee flexion during the injury. The goals ${ }^{[1]}$ of surgical treatment includes the following

- Preservation of knee cap to greatest possible extent

- Restoration of functional integrity and strength of extensor mechanism

- Maximizing articular congruity

- Early mobilization

Classification of Patella fractures

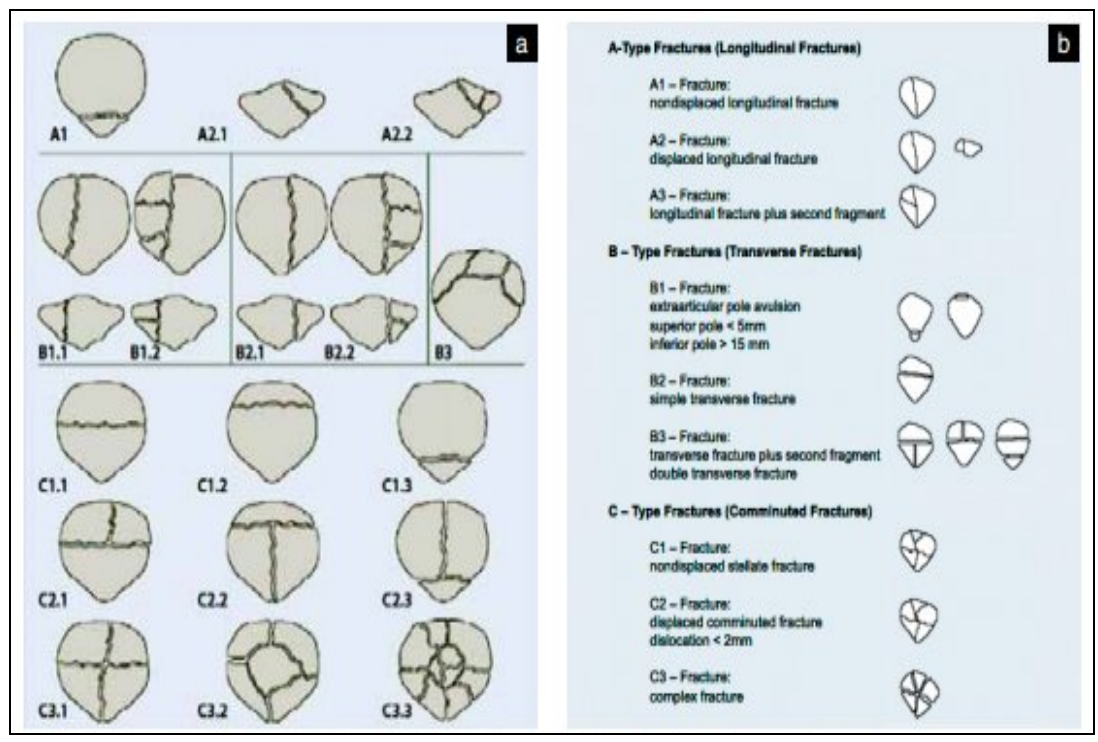

Fig 1: AO classification of patella Fractures

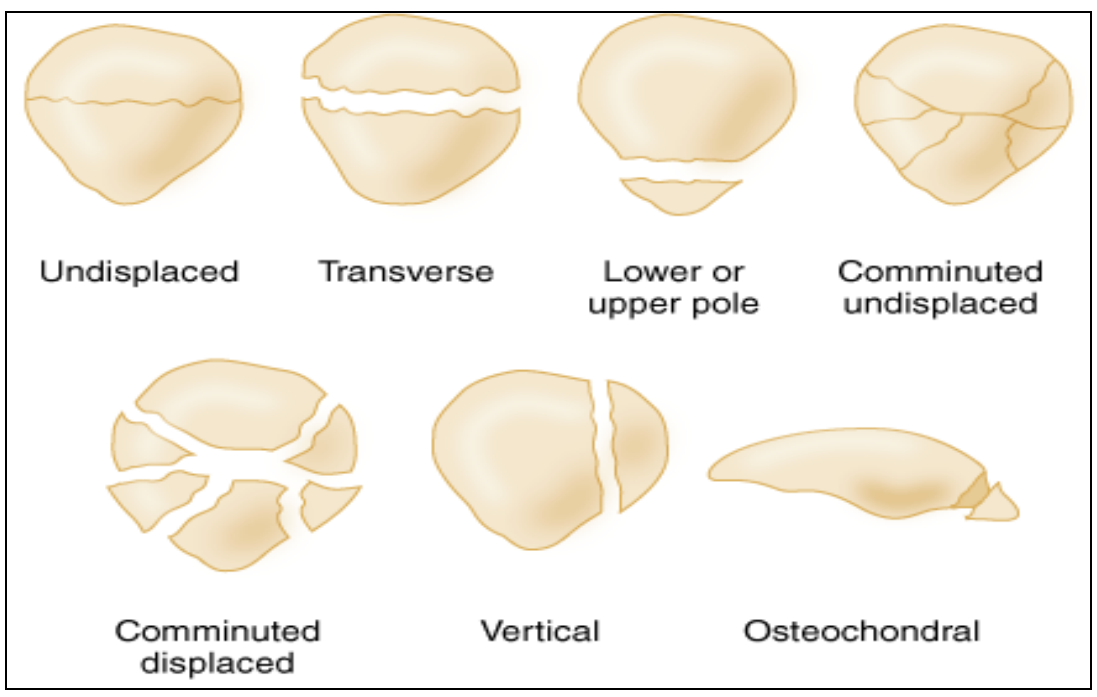

Fig 2: Common patterns of patella fractures

Indication of surgical intervention in fracture patella are displacement of fragments more than 2 to $3 \mathrm{~mm}$, articular incongruity and compound fractures.

Tension band wiring ${ }^{[2]}$ is the treatment of choice performed with large longitudinal incision over the anterior aspect of the knee. Previously this was done by a transverse incision over the anterior aspect of knee which compromises subsequent total knee replacement. Complications associated with open reduction includes infection, cosmetically unsightly scar, delayed wound healing, broken wires, irritation, migration of Kirschner wires, fixation failure, postoperative adhesions, patello femoral arthritis secondary to postoperative articular step, prolonged disability for work. The subsequent revision rate that was reported in various studies were between $7 \%$ and 43\%. These complications were related with open approach, fixation device, or both. But in case of minimally invasive percutaneous technique there will be preserved vascular supply to fracture fragments which accolades to fracture union permitting early rehabilitation and aesthetic scar.

\section{Methodology}

This was a prospective study conducted from March 2015 to December2016 at Department of Orthopedics at Coimbatore medical college hospital. Patients were selected based on the following criteria.

\section{Inclusion criteria}

- Closed and grade 1 open transverse fractures of patella.

- Acute displaced fractures $>2$ to $3 \mathrm{~mm}$

- Articular incongruity $<2 \mathrm{~mm}$ 


\section{Exclusion criteria}

- Comminuted fractures

- Superior and inferior pole fractures, vertical fractures.

- Severe osteopenia

- Injuries more than 10 days

- History of previous knee surgery

- Grade 2 \& 3 Open fractures

- Patients requiring intensive care,

- Bedridden/ non ambulant patients, pregnant women

- Paediatric fractures.

20 cases of transverse fractures of the patella with a displacement $>2 \mathrm{~mm}$ were included in the study. All the patients were evaluated preoperatively by standard AP and Lateral Xray of the knee. All the patients were operated within
5 days of injury. There were fourteen male and six female patients in our study. The age group range from 22 to 50 years. There were 16 closed and 4 grade 1 compound fractures. 11 patients had fractures on right and 9 on the left side. All medical records of the patients were reviewed comprehensively, including injury time, mode of injury, soft tissue injury, associated trauma, operative time, post op complications and postoperative rehabilitation. Follow-up records including the functional recovery, complications, and the need for further surgery were reviewed in detail. Radiographic images were studied regarding initial fracture type, displacement, postoperative reduction, implant position and fracture union. Definitive assessment of functional outcome by using the Lysholm knee scoring rating system was done.

Table 1: Demographic pattern of patella fractures

\begin{tabular}{|c|c|c|c|}
\hline S. No & Variables & \multicolumn{2}{|c|}{} \\
\hline \multirow{3}{*}{1} & \multirow{3}{*}{ Age } & 20 to 30 & 5 \\
\cline { 3 - 4 } & & 30 to 40 & 9 \\
\cline { 3 - 4 } & & 40 to 50 & 6 \\
\hline 2 & Male: female & 14 & 6 \\
\hline 3 & Closed : compound & 16 & 4 \\
\hline 4 & Right : Left & 11 & 9 \\
\hline \multirow{3}{*}{5} & \multirow{3}{*}{ Mode of injury } & Indirect impact & 12 \\
\cline { 3 - 4 } & & Fall from height & 5 \\
\cline { 3 - 4 } & & RTA & 3 \\
\hline
\end{tabular}

\section{Surgical technique}

Surgery was performed under spinal anaesthesia with patient in supine position with knee in flexed position. A stab incision of $1 \mathrm{~cm}$ was made at the supero-lateral border of the patella. An 18 gauge SS wire was then introduced through it and brought out through superomedial border using a wire passer through the quadriceps tendon. The fracture was held reduced by pointed reduction forceps applied subcutaneously. Then through the superomedial portal of the patella SS wire was passed toward its inferolateral portal. The wire was then reintroduced through the inferolateral portal towards the inferomedial portal through the patellar tendon. The wire was introduced through the inferomedial portal toward the superolateral portal subcutaneously over the patella. Both ends tightened, knotted and bent. Reduction was checked under a Carm image intensifier.

Postoperatively long knee brace was used. Passive range of motion was started postoperatively within the tolerable limits of pain. Protected weight bearing with walking fame was allowed at the first postoperative day. Three days after the surgery, patients were discharged. Clinical evaluation and suture removal done at $10^{\text {th }}$ day. Active range of motion was started after 3 weeks of surgery. Full weight-bearing without walking assistance was started after 8 weeks. Subjective (knee pain, stiffness, kneeling discomfort, limitation of activity and weakness) and objective scores were analyzed.
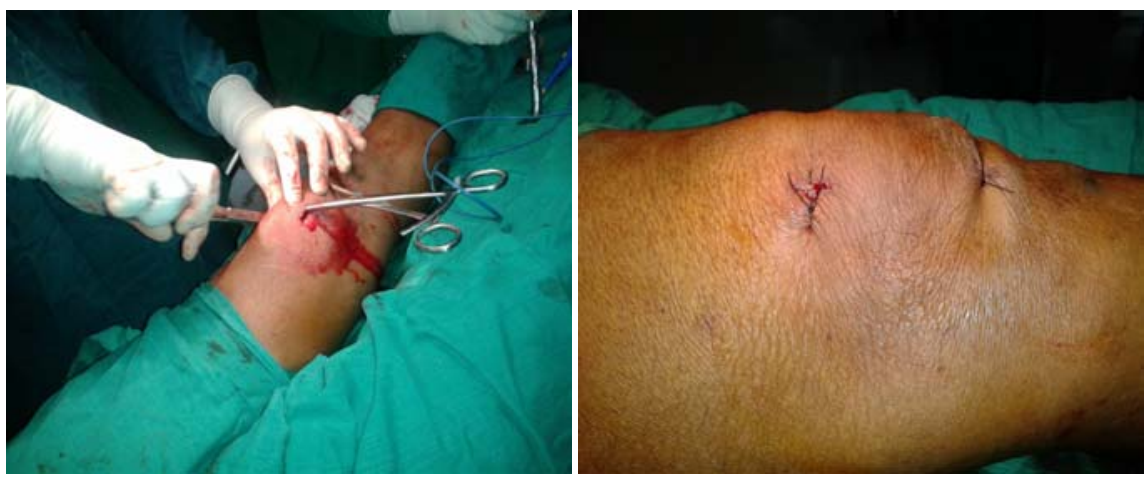

Fig 3: Intra operative images

The patients were followed up for a minimum period for 6 months. During follow- ${ }^{-}$up pain severity was assessed based on Lysholm knee score and time to ambulation was noted along with time to union and any complication.

\section{Results}

Operative time and blood loss in percutaneous tension band wiring is much less compared with open tension band wiring.
This technique both clinically and radiologically showed rapid bony union. Lysholm knee score is more with percutaneous tension band wiring with an average of 92 . Mean operating time was 30 (range 20 to 50) minutes. The mean follow up period was 8 months (range 6 to 12) months. At the latest follow-up, all patients had good results with painless knee motion, no quadriceps wasting and no subjective disability. Total range of motion was excellent at an average of 142.4 
degrees of knee flexion. The objective score was excellent in 14 patients and good in 6 and none had fair or poor scores whereas the subjective score was excellent in 16 , good in 4.There is no extensor lag in any of our cases. There were no complications like infection, limitation of knee motion, loss of reduction, implant breakage. All patients had radiological union at eight weeks.
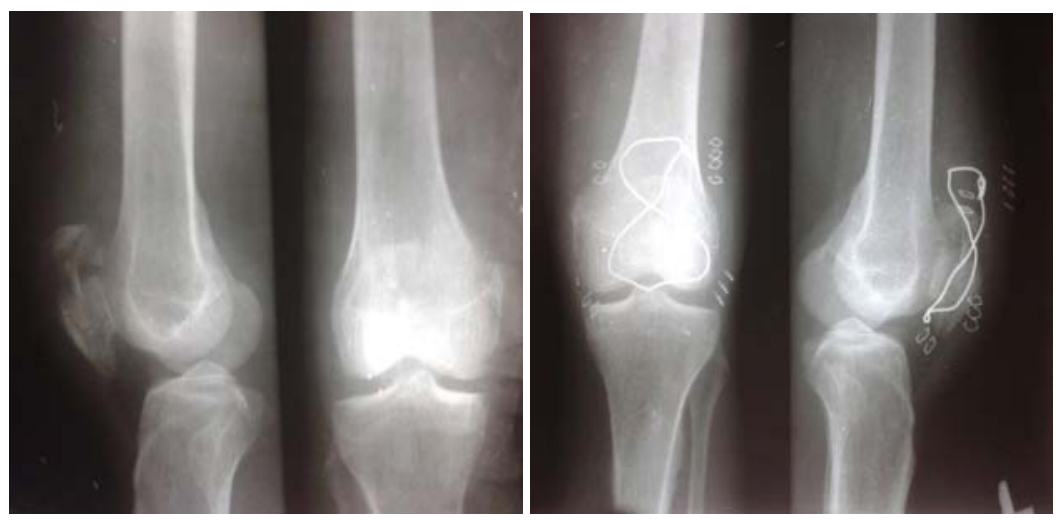

Fig 4: Preop and post op Xray images

Functional outcome was assessed based on Lysholm knee score as in figure 5.

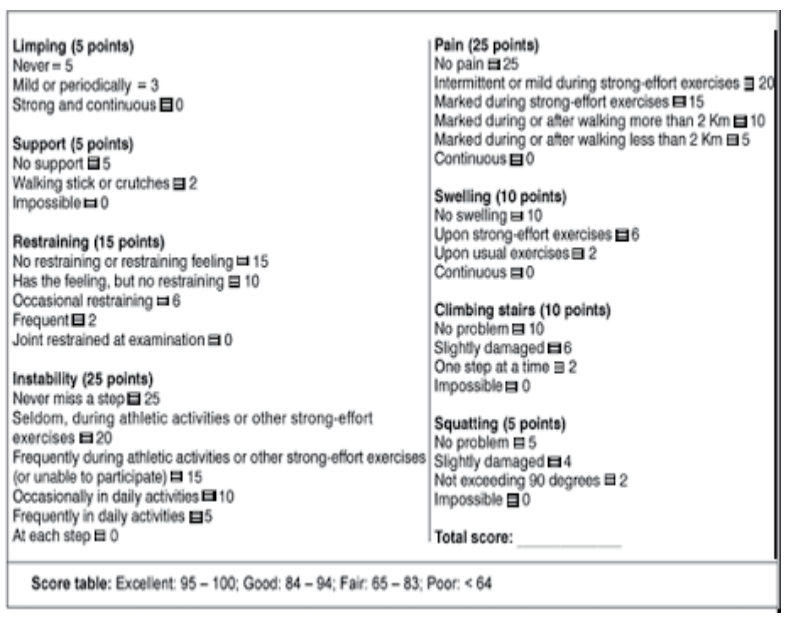

Fig 5: Lysholm Knee score

The final functional outcome of all the patients in our study based on Lysholm knee score is as shown in table 2 .

Table 2: Functional outcome based on Lysholm knee score

\begin{tabular}{|c|c|c|}
\hline Functional outcome & No of cases & Percentage \% \\
\hline Excellent $>90$ & 14 & 70 \\
\hline Good 84 to 90 & 6 & 30 \\
\hline Fair 65 to 83 & 0 & 0 \\
\hline Poor $<65$ & 0 & 0 \\
\hline
\end{tabular}

\section{Discussion}

Fractures of patella are not uncommon, they often result in residual patellofemoral arthralgia, quadriceps weakness, restricted knee motion and degenerative arthritis. The patella provides the critical biomechanical function of both linking and displacement during flexion to extension of the knee. The goals of surgical treatment are to reestablish continuity of extensor mechanism and to restore a smooth articular surface by stable internal fixation to permit early knee motion.

The main treatment options for patellar fractures are the following ${ }^{[3]}$.

1. Non operative management for minimally displaced fractures which consists of extension splinting / bracing for 6 weeks.

2. Open reduction internal fixation such as modified tension band wiring/ percutaneous wiring, cerclage wiring, screw fixation for longitudinal fractures.

3. Partial patellectomy with patellar tendon repair.

4. Complete patellectomy.

The principle of tension band wire fixation of patellar fracture is to convert the tensile forces generated from quadriceps complex to the anterior cortical surface of patella into compressive forces at the articular surface. With progressive knee flexion, the passive tensile forces in the extensor mechanism in addition to the pressure of the femoral condyles against the patella increase inter fragmentary compression at articular surface there by promoting rapid union. Results of our study showed that percutaneous technique could provide satisfactory osteosynthesis, better functional outcome, and fewer complications. Operative time and blood loss in percutaneous tension band wiring is much less. Avoidance of extensive incisions, preservation of blood supply to the patella, lesser requirement of arthrotomy are observed. Percutaneous tension band wiring clinically and radiologically showed rapid bony union. All patients achieved excellent reduction and good functional outcome. Repair of the retinacular tear is recommended for patients who prefer early postoperative motion nonetheless retinacular repair is not a standard procedure for all minimally invasive surgeries and none of our patients have any extensor lag despite having no retinacular repair

According to Biyani et al ${ }^{[4]}$, the most important factor adversely affecting the long term results is the delay in the operation. Besides patient's health status, stabilization of fractures can be delayed because of status of the skin, especially in excessive abrasions and bruises to reduce postoperative complications. He described percutaneous tension band wiring technique for minimally displaced patella fractures using a single SS wire in a figure of 8 construct anterior to patella in subcutaneous plane. Intra osseous stabilization $\mathrm{k}$ wires are not used. The retention of the anterior soft tissue envelope likely to reduce morbidity such as neuroma formation and infection that will adversely affect the functional outcome. The percutaneous technique keeps the soft tissue damage to a minimum that may assist in early rehabilitation. 
Jin ho cho Et al ${ }^{[5]}$ (2013) performed percutaneous tension band wiring for patella fractures using cannulated screws. In this study the average age group was 45 years, in our study is 35. The mean follow up period is 15 months compared to 6 months in our study. The radiographic bone union occurs by 7.3 weeks, in our study it was 8 weeks. The range of knee flexion achieved is 133 degrees compared to 142.4 degrees. The mean Lysholm score is 93 compared to 92 in our study. Tomoji et al ${ }^{[6]}$ (2014) performed percutaneous cerclage wiring in 5 patients in 2 layers, each around superficial and deep layers of patella. In this study the average flexion is 141 degrees, bony union achieved in 4 cases $(80 \%)$, one case had superficial infection and two cases had implant breakage. Patella femoral arthritis was not encountered in this study. Leung et al ${ }^{[7]}$ performed similar percutaneous wiring in a figure of 8 loop over anterior surface of patella and showed excellent result in 5 cases. Ma yz et al ${ }^{[8]}$ did percutaneous sutures for patellar fractures and had poor results in 5 patients and has concluded that neither open reduction nor percutaneous suture with cerclage solves the problem of the tendency of the fracture fragments to gap anteriorly. Appel et al [9] has done a study on successful management of displaced transverse fractures of the patella accomplished by using an arthroscopically assisted technique. Here the morbidity is markedly reduced as the surgery is usually done on same day and recuperation is accelerated.

Makino et al ${ }^{[10]}$ (2002) performed arthroscopic assisted percutaneous tension band wiring for patellar fractures using 2 inter fragmentary screws. Here the reduction was assisted via arthroscope. Matthew et al ${ }^{[11]}$ (2009) performed minimally invasive fixation for minimally displaced patellar fracture using $2 \mathrm{k}$ wires with tension band wire. Akilesh et al ${ }^{[12]}$ (2012) performed percutaneous fixation of 23 cases of transverse patellar fractures with tension band wiring with two parallel $\mathrm{k}$ wires. The mean operating time was 46 minutes, the mean follow up period was 20 months. All patients had radiological union in 8 weeks. One patient had patello femoral arthritis, 2 had superficial infections, 3 had hardware problems in this study. Kulkarni et al [13] (2014) performed percutaneous tension band wiring and encirclage loop for transverse fracture with arthroscopic evaluation of under surface of patella. Turgut et al [14] did percutaneous, arthroscopic-assisted osteosynthesis of patellar fractures associated with skin abrasions and lacerations in 11 patients. Skin problems did not delay the operation and the rehabilitation. The average follow up was 2.8 years. All fractures healed without complications and good results were obtained in all patients. Tandogan et al [15] had concluded from his study that arthroscopicassisted reduction and percutaneous cannulated screw fixation is appropriate for displaced transverse patellar fractures without major separation and comminution. Longitudinal or oblique fractures, even if there are more than 2 major fragments, are amenable to arthroscopic techniques, provided the fragments are large enough to be fixed with screws. This technique is minimally invasive and does not disturb the vascular supply of patella. It allows clear visualization of the reduction and stability of the fracture and facilitates early postoperative range of motion exercises. This method is not suitable for highly comminuted fractures or transverse fractures with major separation that are accompanied by rupture of the extensor mechanism.

El-Sayed et al ${ }^{[16]}$, in his study included fourteen patients with displaced transverse fractures of the patella that were treated by arthroscopic-assisted closed reduction of the fracture with percutaneous screw fixation. The mean period of follow up was 26 months. All fractures united in a mean period of 7 weeks. Twelve patients regained full knee range of motion and two patients had 10 degrees loss of full flexion. The mean final Lysholm score was 93. The mean final lysholm score in our study was 92. Arthroscopic-assisted reduction and percutaneous screw fixation is an appropriate technique for displaced transverse patellar fractures without major separation or comminution. Luna-Pizarro D et al ${ }^{[17]}$ has done a randomized controlled study about management of displaced transverse fractures of patella by using a percutaneous patella osteosynthesis system (PPOS) using Steinmann pins as reduction tool and performed tension band wiring with parallel $\mathrm{k}$ wires and SS wires.

A stable internal fixation with a minimally invasive technique theoretically allows initiation of earlier rehabilitation, which, in turn, can prevent chronic joint pain, joint adhesions, muscular atrophy, posttraumatic arthritis, ankylosis. Drawbacks still existed in our study. Disadvantages of the percutaneous wiring include its technical difficulty, uncertainty about the behavior of the fragments after the reduction and retinacular tear cannot be repaired simultaneously. Our technique is indicated for displaced transverse patellar fractures but not recommended for more comminuted fractures with multiple displaced fragments with articular step or incongruity. For these complicated fractures, precarious reduction by open method is recommended.

\section{Conclusion}

Patellar fractures managed by percutaneous tension band wiring provides better functional outcome. Early postoperative rehabilitation can be achieved with this technique. This method could be a better alternative to open surgery especially in displaced transverse fracture of the patella. Our procedure is less time consuming and simple. To perform this procedure no extraordinary skill is required.

\section{References}

1. Patella Fractures. Extensor Mechanism Injuries, Asheesh Bedi Madhav A, Karunakarni Rockwood. And Green's Fractures In Adults, 7th Edition

2. Hung LK, Chan KM, Chow YN, Leung PC. Fractured patella: operative treatment using the tension band principle. Injury. 1985; 16:343-7

3. Weber MJ, Janecki CJ, McLeod P, Nelson CL, Thompson JA. Efficacy of various forms of fixation of transverse fractures of the patella. J Bone Joint Surg Am. 1980; 62:215-20.

4. Biyani A, Mathur NC, Sharma JC. Percutaneous tension band wiring for minimally displaced fractures of the patella. International Orthopaedics. 1990; 14:281-3

5. Jin-Ho Cho et al. Percutaneous Cannulated Screws with Tension Band Wiring Technique in Patella fractures, Knee Surg Relat Res. 2013; 25(4):215-219.

6. Tomoji Matsuo et al. Percutaneous cerclage wiring for the surgical treatment of displaced patella fractures. Strat Traum Limb Recon. 2014; 9:19-23.

7. Leung PC, Mak KH, Lee SY. Percutaneous tension band wiring: a new method of internal fixation for mildly displaced patella fracture. J Trauma. 1983; 23:62-4.

8. Ma YZ, Zhang YF, Qu KF, Yeh YC. Treatment of fractures of the patella with percutaneous suture. Clin Orthop Relat Res. 1984; 191:235-41

9. Appel MH, Seigel H. Treatment of transverse fractures of the patella by arthroscopic percutaneous pinning. Arthroscopy. 1993; 9:119-21. 
10. Makino et al. arthroscopic assisted surgical technique for treating patellar fractures. Journal of arthroscopy and related surgeries. 2002: 18(6):671-675.

11. Matthew Hall J. Minimally invasive technique for fixation of minimally displaced patellar fracture, Injury Extra 40, 2009, 74-76

12. Akhilesh Rathi et al. Percutaneous tension band wiring for patellar Fractures Journal of Orthopaedic Surgery. 2012; 20(2):166-9.

13. Kulkarni PG, Dudhwala Utkal. Percutaneous Tension Band Wiring \& Encirclage loop for Transverse fracture of Patella, Journal of Trauma \& Orthopaedics. 2014; 9(4):21-22.

14. Turgut A, Gunal I, Acar S, Seber S, Gokturk E. Arthroscopic-assisted percutaneous stabilization of patellar fractures. Clin Orthop Relat Res. 2001; 389:57-61.

15. Tandogan RN, Demirors H, Tuncay CI, Cesur N, Hersekli M. Arthroscopic-assisted percutaneous screw fixation of select patellar fractures. Arthroscopy. 2002; 18: 156-62.

16. El-Sayed AM, Ragab RK. Arthroscopic-assisted reduction and stabilization of transverse fractures of the patella. Knee. 2009; 16:54-7.

17. Luna-Pizarro D, Amato D, Arellano F, Hernandez A, Lopez-Rojas P. Comparison of a technique using a new percutaneous osteosynthesis device with conventional open surgery for displaced patella fractures in a randomized controlled trial. J Orthopaedic Trauma. 2006; 20:529-35. 